\title{
Predictive Elution Window Stretching and Shifting as a Generic Search Strategy for Automated Method Development for Liquid Chromatography
}

Eva Tyteca, ${ }^{\dagger}$ Anuschka Liekens, ${ }^{\dagger}$ David Clicq, ${ }^{\ddagger}$ Ameriga Fanigliulo, ${ }^{\S}$ Benjamin Debrus, $"$ Serge Rudaz, ${ }^{\|}$ Davy Guillarme," and Gert Desmet* ${ }^{\dagger}$

${ }^{\dagger}$ Vrije Universiteit Brussel, Department of Chemical Engineering (CHIS-IR), Pleinlaan 2, 1050 Brussels, Belgium

${ }^{\ddagger}$ UCB Pharma, Analytical Development Chemicals, Chemin du Foriest, 1420 Braine L'alleud, Belgium

${ }^{\S}$ Aptuit, via Fleming 4, 37135 Verona, Italy

"University of Geneva, University of Lausanne, School of Pharmaceutical Sciences, Boulevard d'Yvoy 20, 1211 Geneva 4, Switzerland

\section{Supporting Information}

ABSTRACT: We report on the possibilities of a new method development (MD) algorithm that searches the chromatographic parameter space by systematically shifting and stretching the elution window over different parts of the time-axis. In this way, the search automatically focuses on the most promising areas of the solution space. Since only the retention properties of the first and last eluting compounds of the sample need to be (approximately) known, the algorithm can be directly applied to samples with unknown composition, and the proposed solutions are not sensitive to any modeling errors. The search efficiency of the algorithm has been evaluated on an extensive set of random-generated in silico samples covering a broad range of different retention properties. Compared to a pure grid-based search, the algorithm could reduce the number of missed components by $50 \%$ and more. The algorithm has also been applied to solve three different real-world separation problems from the pharmaceutical industry. All problems could be successfully solved in a very short time (order of 12 $\mathrm{h}$ of instrument time).

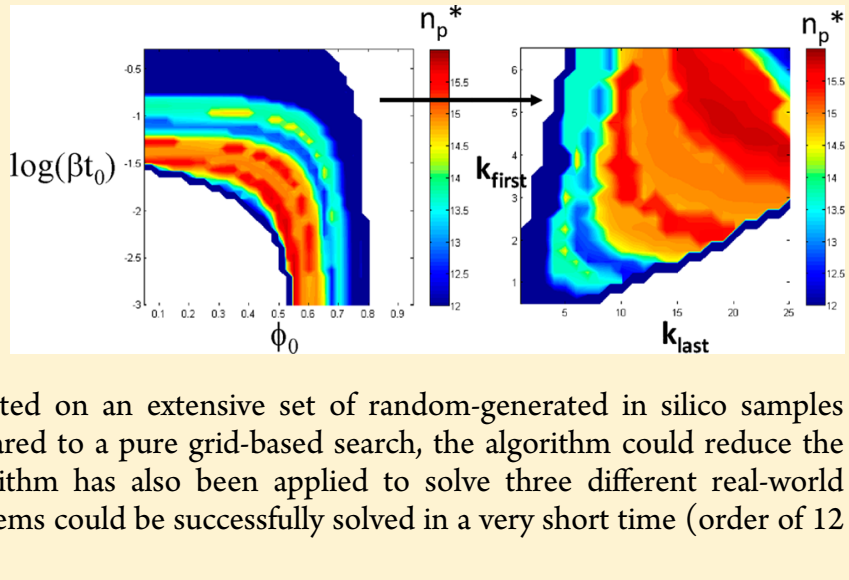

$\mathrm{O}$ ne of the most time-consuming tasks in analytical liquid chromatography is method development (MD). This is the search for the chromatographic operating conditions (type of organic modifier and stationary phase, temperature, gradient profile, $\mathrm{pH}$, ionic strength, etc.) leading to the complete resolution of the sample in all its individual constituents. ${ }^{1-9}$ Because of the high probability for peak overlap ${ }^{10}$ and the sensitive dependency of the retention time of the individual analytes on, for example, the employed gradient slope, the MD process still involves a lot of trial and error and can take up to several weeks of work.

To speed up this work, fully or semiautomated MD software has been developed over the years. ${ }^{10-16}$ Roughly spoken, the automated $\mathrm{MD}$ strategies described in literature are either search-based (e.g., using the Simplex method) ${ }^{17}$ or modelbased (e.g., Drylab, ${ }^{18}$ Chromsword) ${ }^{19}$ In the present study, the properties of a hybrid method, further referred to as the predictive elution window stretching and shifting method $\left(\mathrm{PEWS}^{2}\right.$ ), were investigated. This method explores the chromatographic parameter space in a pure search-mode but also uses the information on the retention properties of some of the peaks (e.g., the first and the last peak of the chromatogram and/or the first and last peaks of its most problematic zone). This information is used to make a model-based prediction of the gradient slope (expressed here as $\left.\beta t_{0}=\left(\phi_{\mathrm{e}}-\phi_{0}\right) \cdot t_{0} / t_{\mathrm{G}}\right)$ and initial gradient composition $\phi_{0}$ values that should be imposed to shift and stretch the elution window of the sample in a controlled manner over different parts of the time-axis. Hence, instead of optimizing the gradient by searching in the $\left(\phi_{0}, \beta t_{0}\right)$ space, the PEWS ${ }^{2}$-method searches directly in the $\left(k_{\text {first }}, k_{\text {last }}\right)$ space. Since the values of $k_{\text {first }}$ and $k_{\text {last }}$ directly determine how wide the elution window is and whether the components elute early or late, these parameters control the peak capacity and selectivity of the chromatogram in a much more direct way than the $\phi_{0}$ and $\beta t_{0}$ parameters.

To test the PEWS ${ }^{2}$-method, it has been applied to a number of different pharmaceutical MD problems. To generalize the results, the search efficiency of the PEWS ${ }^{2}$-method has also been evaluated in a comprehensive numerical test, involving hundreds of different random in-silico samples with widely differing retention properties. In this numerical part of the study, the PEWS ${ }^{2}$-method is compared to that of a direct search in the $\left(\phi_{0}, \beta t_{0}\right)$-space using the most simple of all possible

Received: May 31, 2012

Accepted: August 19, 2012

Published: August 20, 2012 
search algorithms, i.e., using a simple grid search. The latter was selected to minimize the influence of the employed search algorithm itself. In a next step, the PEWS ${ }^{2}$-method can be extended with more advanced search methods, for example, by making use of the information on the local slope of the chromatographic response surface. The method can potentially also be applied to enhance the optimization of multisegment gradients. $^{20-23}$

All searches conducted in the present study were guided using a so-called chromatographic response function or CRF. ${ }^{12,16,20,24-30}$ The CRF used in the present study was defined as the sum $\left(n_{\mathrm{p}}{ }^{*}\right)$ of the number of observed peaks $\left(n_{\mathrm{p}}\right)$ and peak shoulders $\left(n_{\mathrm{s}}\right)$ plus a so-called noninteger part (NIP), describing the quality of the separation:

$$
n_{\mathrm{p}}^{*}=n_{\mathrm{p}}+n_{\mathrm{s}}+\mathrm{NIP}
$$

$$
\text { with NIP }=\sqrt[a n_{\text {crit }}]{\prod_{i} \frac{f_{i}}{g_{i}}}+b \frac{\sum \frac{f_{i}}{g_{i}}}{n_{\text {crit }}}
$$

In this expression, $f_{i} / g_{i}$ is the ratio between the depth of the valley $f_{i}$ and the interpolated peak height $g_{i}$ and therefore only varies between 0 and $1,{ }^{26}$ whereas $n_{\text {crit }}$ is the number of critical pairs (i.e., the number of peak pairs for which $f_{i} / g_{i}<1$ ). When a shoulder is detected, it is counted as an extra peak with an $f_{i} / g_{i-}$ value of 0.01 . By choosing the value of the weighing factors $a$ and $b$ such that their sum maximally equals 0.99 , the NIP-part can also never exceed this value (NIP $\leq 0.99)$ because the factors appearing in the two terms have been normalized by respectively taking the $\left(n_{\text {crit }}\right)^{\text {th }}$ root and by dividing by $n_{\text {crit }}$. This implies that conditions revealing a new compound automatically get a higher $n_{\mathrm{p}}{ }^{*}$-value than conditions leading to a fully baseline resolved separation but revealing one peak less. In the present study, the values of $a$ and $b$ were taken equal (with $a+$ $b=0.99$ ), but many different variants can be proposed (for example, by taking $a=0, b=1$ or vice versa).

\section{EXPERIMENTAL AND NUMERICAL METHODS}

Numerical Methods. Chromatogram Simulation. All numerical simulations were conducted assuming that the elution properties of the compounds can be described by the linear solvent strength (LSS)-model. ${ }^{31-33}$ This was purely done for the sake of simplicity, since there is no reason to assume that the main conclusions of the present study would be different under non-LSS conditions.

In the LSS-model, the retention properties of a compound are fully described by a $k_{w^{-}}$and $S$-value. To simulate a chromatogram containing $n_{\mathrm{c}}$ compounds, a random number generator was used to randomly pick $n_{\mathrm{c}}$ combinations of $k_{\mathrm{w}}$ and $S$-values taken from a given interval. Also the injected concentration of each component was randomly picked from a prescribed interval. The reader is referred to Table $S 1$ in the Supporting Information for a list of the employed intervals.

These values, together with a given value of the column efficiency $N$ and the gradient parameters $\phi_{0}$ (initial mobile phase concentration) and $\beta t_{0}$ (gradient steepness $\beta=\left(\phi_{\mathrm{e}}-\right.$ $\left.\left.\phi_{0}\right) \cdot t_{0} / t_{\mathrm{G}}\right)$ were subsequently used in a computer program written in Matlab to simulate the expected chromatograms. Again for the sake of simplicity, the concentration profiles of the different analyte peaks were assumed to be purely Gaussian, such that their variation with the time $t$ can be written as ${ }^{34,35}$

$$
\begin{gathered}
c\left(\frac{t}{t_{0}}\right)=\frac{c_{0} \sqrt{N}}{\left(1+k_{\mathrm{e}}\right) G} \exp \left(\frac{-N\left(\frac{t}{t_{0}}-1-k\right)^{2}}{2\left(1+k_{\mathrm{e}}\right)^{2} G^{2}}\right) \\
\text { with } c_{0}=\frac{M}{\sqrt{2 \pi} V_{0}}
\end{gathered}
$$

where $t$ is the time ( $\mathrm{min}), t_{0}$ the column dead time $(\mathrm{min}), c_{0}$ the injected concentration $(\mathrm{g} / \mathrm{mL}), k_{\mathrm{e}}$ the retention factor experienced by a compound at the end of the column, $k$ the effective retention factor of a compound ( $k$ is given by $k=t_{\mathrm{R}}-$ $t_{0} / t_{0}$ wherein $t_{\mathrm{R}}$ is the retention time of the compound), $G$ the gradient compression factor, ${ }^{36} M$ the injected mass $(\mathrm{g})$, and $V_{0}$ the column dead volume $(\mathrm{mL})$.

With the known values of $k_{\mathrm{w}}$ and $S$, the values of $k_{\mathrm{e}}$ and $k$ have been calculated for each compound as a function of $\phi_{0}$ and $\beta t_{0}$ using the expressions proposed by Schoenmakers et al. $^{33}$

Actual Search Algorithm. The actual search algorithm starts by determining the retention parameters of two (or more) of the most easily identifiable or problematic peaks in the chromatogram. These can be any of the peaks, but for the sake of argument and as an illustrative example, most of the examples in the present study focus on the first and the last peak of the chromatogram. The retention parameters were determined using the measured retention factor $k$ of the peaks of interest and solving the well-established LSS-expressions for $k^{33}$ using the known values $\beta t_{0}$ and $\phi_{0}$ via the Solver-routine in MS Excel:

Regime 1:

$$
\begin{aligned}
& t_{0}<t_{\mathrm{R}}<t_{0}+t_{\mathrm{D}} \\
& k_{\text {effective }}=k_{0}
\end{aligned}
$$

Regime 2:

$$
\begin{aligned}
& t_{0}+t_{\mathrm{D}}<t_{\mathrm{R}}<t_{0}+t_{\mathrm{D}}+t_{\mathrm{G}} \\
& k_{\text {effective }}=\frac{t_{\mathrm{D}}}{t_{0}}+\frac{1}{b} \ln \left(\left(t_{0}-\frac{t_{\mathrm{D}}}{k_{0}}\right) \frac{b}{t_{0}} k_{0}+1\right)
\end{aligned}
$$

Regime 3:

$$
\begin{aligned}
& t_{\mathrm{R}}>t_{0}+t_{\mathrm{D}}+t_{\mathrm{G}} \\
& k_{\text {effective }}=k_{\mathrm{e}}-\frac{k_{\mathrm{e}}}{k_{0}} \frac{t_{\mathrm{D}}}{t_{0}}+\frac{t_{\mathrm{D}}+t_{\mathrm{G}}}{t_{0}}+\frac{k_{\mathrm{e}}}{k_{0}} \frac{1}{b}\left(1-\exp \left(b \frac{t_{\mathrm{G}}}{t_{0}}\right)\right) \\
& \text { where } k_{\mathrm{e}}=k_{\mathrm{w}} \exp \left(-S \varphi_{\mathrm{e}}\right)
\end{aligned}
$$

Equations $4 a-4 c$ are written in such a way that they take the effect of the system dwell time $t_{\mathrm{D}}$ into account. The $k$-values were measured for two different values of $t_{\mathrm{G}}\left(\right.$ and/or $\left.\phi_{0}\right)$ in order to obtain a closed set of two unknowns and two equations (the two measured $k$-values).

Subsequently, the known values of $S$ and $k_{\mathrm{w}}$ are used to resolve eqs $4 \mathrm{a}-4 \mathrm{c}$ to find the $t_{\mathrm{G}^{-}}$(incorporated in $\beta t_{0}$ ) and $\phi_{0^{-}}$ values needed to put the peaks of interest as close as possible to the target $k$-values. In the algorithm, these target values are selected such that they cover the entire solution space as broadly as possible (cf. the black stars in Figure $1 \mathrm{~b}$ as an example wherein 13 different couples of desired $k$-values for the 


\section{(a)

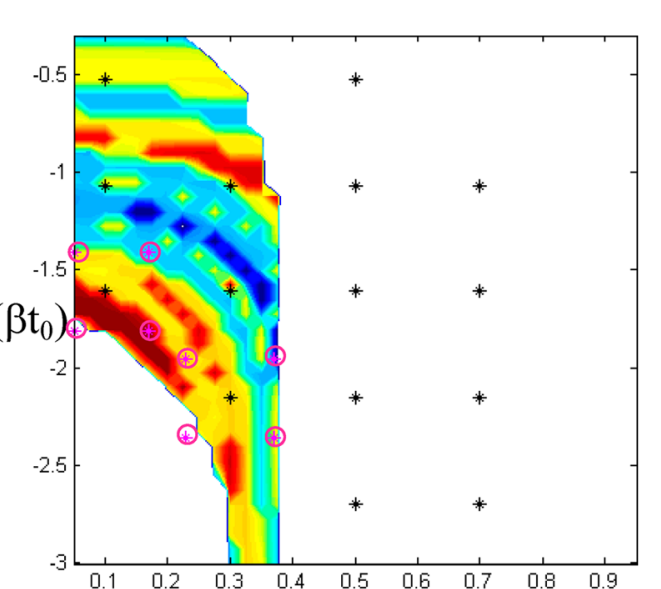

(b)

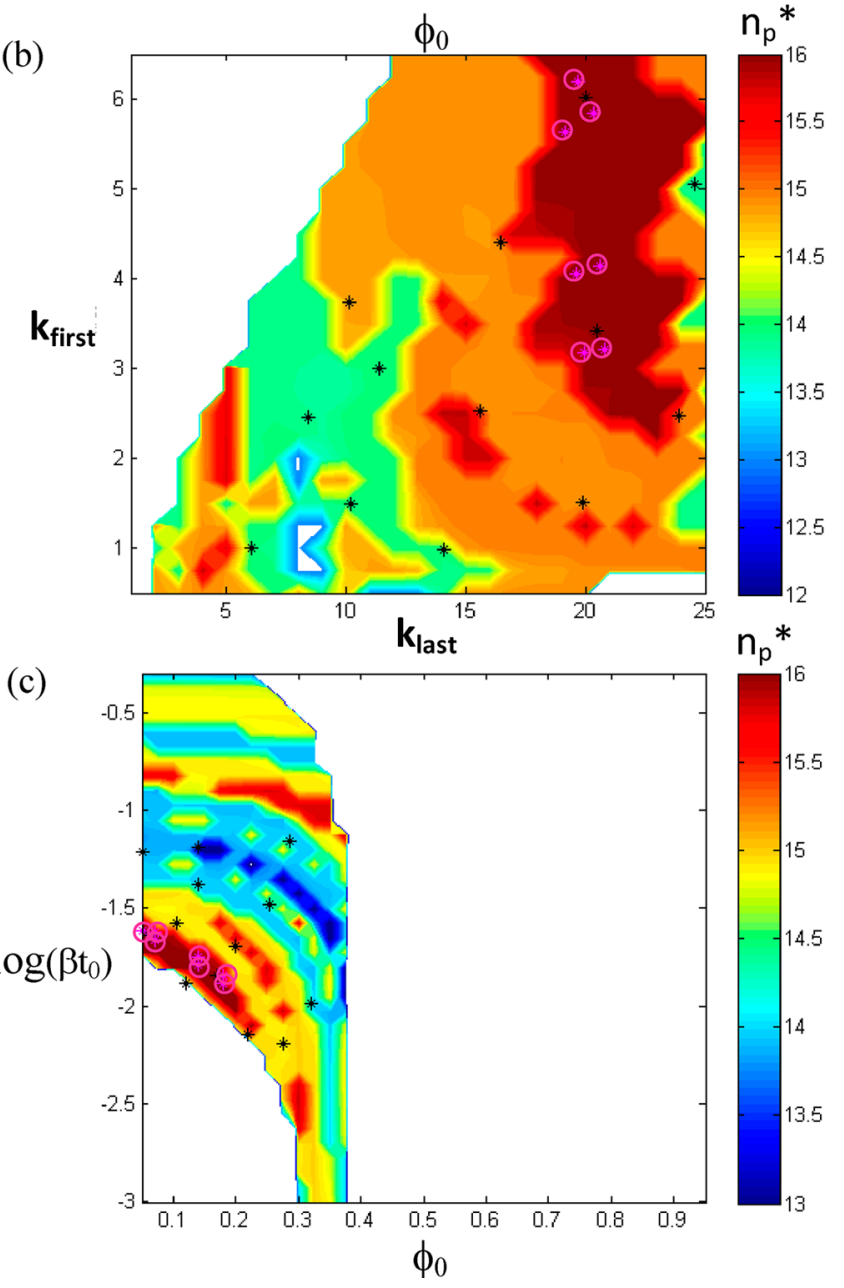

Figure 1. (a) Example of the $\left(\phi_{0}, \beta t_{0}\right)$-contour plot and runs considered in the $\left(\phi_{0}, \beta t_{0}\right)$-grid search for one of the considered 15 component samples. (b) Corresponding $\left(k_{\text {first }}^{\prime} k_{\text {last }}^{\prime}\right)$-contour plot and runs proposed by the PEWS ${ }^{2}$-method. (c) Same runs as in part b but now represented in the $\left(\phi_{0}, \beta t_{0}\right)$-space. Legend: $*$, initial search; red $*$, fine-tuning steps.

first and last peak in the chromatogram were selected). Typically, the Excel solver finds a solution in less than $1 \mathrm{~min}$.

After the execution of the runs corresponding to each of the different target value combinations (cf. again the 13 black star data points in Figure $1 \mathrm{~b}$ ), the separation quality of each of the obtained chromatograms is calculated using the CRF- expression given by eqs 1 and 2 . Subsequently, some additional fine-tuning runs are conducted around the $k$-values of the runs with the highest CRF.

Columns, Reagents, and Instruments. Samples. The PEWS $^{2}$-method has also been tested by applying it to a number of real-world MD problems. The first sample consisted of an antiepileptic drug (API) and its 13 impurities. The different compounds were diluted in $90 / 10 \mathrm{H}_{2} \mathrm{O} / \mathrm{MeOH}$ to a concentration $1 \mathrm{mg} / \mathrm{mL}$ (API) and $0.2 \mathrm{mg} / \mathrm{mL}$ (9 impurities). To develop a method with Drylab (Molnár Institute, Berlin, Germany), the different compounds have also been prepared separately. A second mixture, containing four additional impurities, has been included with a concentration of 200 $\mathrm{mg} / \mathrm{mL}$. The injection volume was $2 \mu \mathrm{L}$, and the detection wavelength was $258 \mathrm{~nm}$.

In a second example, a method was developed for the separation of a new drug under development (API) and its 13 impurities (synthesis intermediates, synthesis side-products, and degradation impurities). The compounds had a MW between 131 and $335 \mathrm{Da}$ with varying polarity due to different functional groups. The sample was dissolved in $50 / 50 \mathrm{H}_{2} \mathrm{O}$ / $\mathrm{ACN}+0.05 \%$ TFA. The injection volume was $20 \mu \mathrm{L}$. UV detection was carried out at $275 \mathrm{~nm}$.

In a third example, a method has been developed for a 16 antipsychotic drugs sample, consisting of Maprotiline, Duloxetine, Chlorpromazine. $\mathrm{HCl}$, Levomepromazine, Clotiapine-base, Thioridazine, Fluphenazine $2 \mathrm{HCl}$, Aripiprazole, Quetiapine, fumarate, Olanzapine, Chlorprothixen $\cdot \mathrm{HCl}$, Clozapine, Pipamperone, Brotizolam, Flupentixol $2 \mathrm{HCl}$ (both $\mathrm{E}$ and $\mathrm{Z}$ isomer). These were dissolved in $70 / 30 \mathrm{H}_{2} \mathrm{O} / \mathrm{MeOH}$ to a final total concentration ranging between 10 and $40 \mathrm{ppm}$ for all the individual compounds. The injected sample volume was equal to $2 \mu \mathrm{L}$. UV-detection occurred at a wavelength of $230 \mathrm{~nm}$.

Instruments and Columns. For the separation of the antieliptic drug and its impurities, a binary Waters UPLC system (with a dwell volume of $120 \mu \mathrm{L}$ ) has been used. Two Waters Acquity HSS columns $(2.1 \mathrm{~mm} \times 100 \mathrm{~mm}, 1.8 \mu \mathrm{m})$ have been used for the screening: a T3 and a PFP column. The column temperature was set at $30{ }^{\circ} \mathrm{C}$. The mobile phases were $\mathrm{H}_{2} \mathrm{O}+0.1 \%$ TFA and ACN $+0.1 \%$ TFA, at a flow rate of 0.400 $\mathrm{mL} / \mathrm{min}$.

The separation of the 13 components mixture was done on an Agilent 1100 instrument (with a dwell volume of $1.15 \mathrm{~mL}$ ). An Achrom ACE 3 C18 AR column $(150 \mathrm{~mm} \times 4.6 \mathrm{~mm}, 3$ $\mu \mathrm{m})$ was used at $40{ }^{\circ} \mathrm{C}$. The two mobile phases used for the gradient elution were an aqueous buffer (ammonium acetate 20 $\mathrm{mM} \mathrm{pH} \mathrm{5.5)} \mathrm{and} \mathrm{ACN.} \mathrm{The} \mathrm{flow} \mathrm{rate} \mathrm{was} 1 \mathrm{~mL} / \mathrm{min}$.

The 16 antipsychotic drugs sample was separated on an Acquity UPLC H-Class instrument (with a dwell volume of 370 $\mu \mathrm{L})$. Four Waters Acquity columns $(50 \mathrm{~mm} \times 2.1 \mathrm{~mm} 1.7 \mu \mathrm{m})$ have been used for the screening: BEH C18, BEH Shield RP18, CSH Fluoro-Phenyl, and BEH Phenyl. The mobile phases were an aqueous buffer (ammonium formate or acetate $20 \mathrm{mM}$ at $\mathrm{pH} \mathrm{3,5}$, and 9) and ACN. The flow rate was $500 \mu \mathrm{L} / \mathrm{min}$, and the columns were operated at a temperature of $45{ }^{\circ} \mathrm{C}$.

\section{RESULTS AND DISCUSSION}

Illustrative Example (Numerical Simulation). A large part of the development and the optimization of the PEWS ${ }^{2}$ method was done using simulated chromatograms. A simulation study has the advantage that a large number of cases can be tested in a short time and that the $k_{\mathrm{w}}$ - and $S$-values of the individual compounds are always exactly known. As a 
consequence, it can also be exactly calculated how the optimization goal (i.e., the separation quality number $n_{\mathrm{p}}{ }^{*}$ ) varies as a function of the chromatographic variables $\phi_{0}$ and $\beta t_{0}$. In the present study, this information has been used to visualize the efficacy of the PEWS ${ }^{2}$-method by plotting its search steps against a background formed by a high-resolution contour plot of the value of the optimization goal $n_{\mathrm{p}}{ }^{*}$.

Figure la shows an example of such a contour plot $\left(n_{\mathrm{p}}{ }^{*}\right.$ versus $\varphi_{0}$ and $\left.\beta t_{0}\right)$ for the case of a 15 compound mixture. The brown region in the contour map corresponds to the case with the highest separation resolution. The black star data points represent the search grid used for the pure grid-based search in the $\left(\phi_{0}, \beta t_{0}\right)$-space. The number of data points $(15)$ was more or less arbitrarily selected for its ability to cover the space in a sufficiently uniform and dense way without needing an excessive amount of runs (assuming a $30 \mathrm{~min}$ run time including column regeneration, these 15 runs can be completed in $15 \times 30 \mathrm{~min}=7.5 \mathrm{~h}$ of separation run time). The data points at the extremities were left out of the grid search, as these conditions are seldom of importance in reality either.

Figure $1 \mathrm{~b}$ shows the $n_{\mathrm{p}}{ }^{*}$-contour plot of the same sample but now in a coordinate system formed by the retention coefficient of the first and the last eluting compound ( $y$ - and $x$-axis, respectively). Important to note is that the new search grid (black star data points) is now directly fitted onto the $\left(k_{\text {first }}, k_{\text {last }}\right)$-domain. Since these grid points correspond to a controlled shifting and stretching of the elution window, they directly represent the physical meaning of the PEWS ${ }^{2}$-method.

To conduct the search, the linear gradient parameters $\left(\varphi_{0}\right.$ and $\left.\beta t_{0}\right)$ corresponding to each of the black star data points in the $\left(k_{\text {first }} k_{\text {last }}\right)$-domain are calculated using the $k_{\mathrm{w}}$ - and $S$-values of the first and last eluting compound collected during two initial scouting runs with different values for $t_{\mathrm{G}}$ and running between 5 and $95 \%$ of organic modifier and feeding these data to the well-established LSS-model equations for the effective retention coefficient ${ }^{31}$ and use an iterative search algorithm to find the $\beta t_{0}$ and $\phi_{0}$-values yielding the best agreement with the $\left(k_{\text {first }} k_{\text {last }}\right)$-combination of the grid point under consideration. In some cases, the $k_{\text {first }}{ }^{-}$and $k_{\text {last }}$-values of the obtained chromatogram deviated significantly from the expected value. This occurred when the identity of the peaks eluting first and or last changed from one $\left(\phi_{0}, \beta t_{0}\right)$-combination to another. To tackle this problem, a limited "peak tracking" strategy was implemented wherein the $k_{w^{-}}$and $S$-parameters of the three first and the three last eluting compounds were continuously updated by scanning through all possible elution order combinations and taking the one that is most consistent with the observed elution pattern. In the very limited number of cases, wherein also this peak tracking failed, the algorithm still returned a very useful set of $\beta t_{0}$ and $\phi_{0}$-conditions. Although leading to a wrong value for $k_{\text {first }}$ and $k_{\text {last }}$ the resulting chromatograms contained useful information to proceed with the search.

To illustrate the efficacy of the PEWS ${ }^{2}$-method, Figure 1c shows the position of the runs proposed by the PEWS ${ }^{2}$ algorithm but now plotted in the $\left(\phi_{0}, \beta t_{0}\right)$-space. As can be noted, these runs are much better concentrated around the area(s) of maximal resolution than the runs corresponding to the $\left(\phi_{0}, \beta t_{0}\right)$-grid search considered in Figure 1a.

As a consequence, also the additional refinement runs (represented by the pink stars, configured in a square around the two best first round runs in either the $\left(\phi_{0}, \beta t_{0}\right)$-space or the $\left(k_{\text {first }}, k_{\text {last }}\right)$-space $)$ are much better targeted toward the optimal solution region in the PEWS ${ }^{2}$-method (Figure $1 \mathrm{~b}, \mathrm{c}$ ) than in the $\left(\phi_{0}, \beta t_{0}\right)$-grid search. Obviously, in the present example, the search in the $\left(\phi_{0}, \beta t_{0}\right)$-domain also yielded a good solution (see pink grid point close to the contour plot maximum in Figure 1a). However, the probability that this solution was found is clearly much smaller than for the search in the $\left(k_{\text {first }}, k_{\text {last }}\right)$-space, where the number of runs proposed by the algorithm close to the contour plot maximum is much larger, as in total 9 of the proposed runs are conducted in the (brown) region of maximal separation resolution. The high number of runs conducted there also provides important information concerning the robustness of the final method. A second example of a fine search is shown in the Supporting Information (Figure S-1).

Comprehensive Numerical Comparison Test. To quantify the potential advantage of the PEWS $^{2}$-method for a statistically relevant number of samples of different complexity, the method as described in Illustrative Example (Numerical Simulation) has been applied to a large number of in silico samples. Five sample categories were considered, respectively, containing 5, 10, 15, 20, and 25 components. For each category, 200 different samples were generated using the random number generator to pick the $k_{\mathrm{w}^{-}}$and $S$-values from the different considered allowable intervals (see Table S-1 in the Supporting Information). For each sample, a fine and a crude grid search were conducted. The fine search consisted of 15 initial runs, followed by $2 \times 4$ refinement runs (conducted around the two best solutions obtained in the initial round). The crude search only consisted of 7 initial runs. No additional refinement runs were performed. Two examples of a crude search are shown in the Supporting Information (Figures S-2 and S-3).

Figure 2 compares the number of missed components. As can be noted, the PEWS $^{2}$-method consistently leads to a

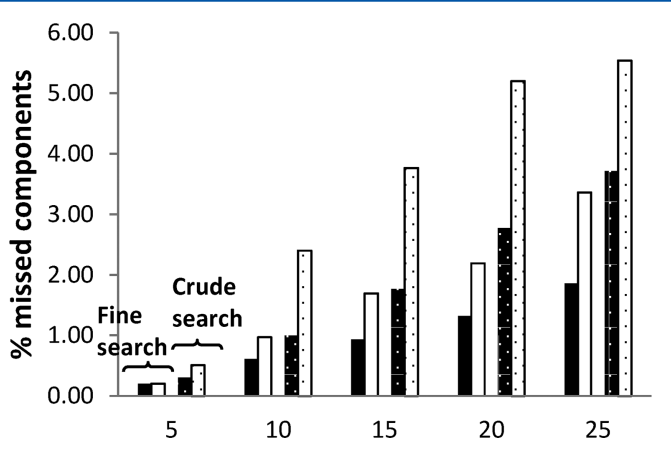

Figure 2. Side-by-side comparison of the PEWS ${ }^{2}$-method (black for fine search; black with white dots for crude search) and the $\left(\phi_{0}, \beta t_{0}\right)$ grid search (white for fine search; white with black dots for crude search) of the number of missed components of the best separations obtained for each of the 200 considered samples considered per sample difficulty (represented by the different number of compounds contained within them: 5, 10, 15, 20, and 25).

smaller percentage of missed components. The higher the number of compounds per sample, i.e., the more demanding the separation, the larger the gain margin of the $\mathrm{PEWS}^{2}$ method with respect to the pure $\left(\phi_{0}, \beta t_{0}\right)$-grid search. This is in agreement with the intuitive expectation that the extra modeling information (i.e., the knowledge of the retention behavior of the first and last eluting compounds) builds into the PEWS $^{2}$-method and becomes more useful when the search is more difficult. The latter can also be noted by comparing the results of the fine grid search with that of the crude grid search. The latter search conditions are more demanding, explaining 


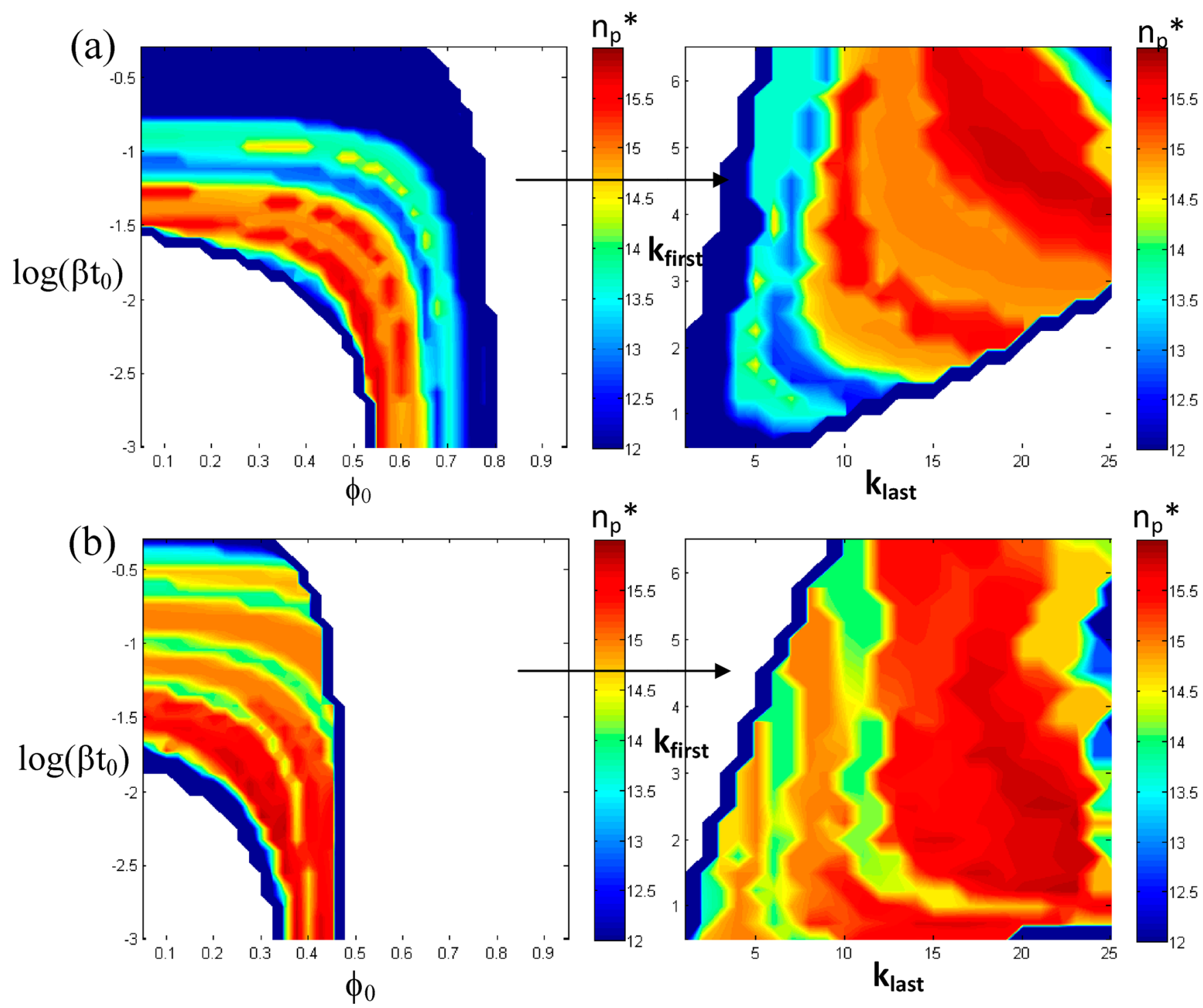

Figure 3. (a,b) Side by side comparison of $n_{\mathrm{p}}{ }^{*}$-contour plots in $\left(\phi_{0}, \beta t_{0}\right)$-space and $\left(k_{\text {first }}, k_{\text {last }}\right)$-space ( 15 component mixture).

why the advantage of the PEWS ${ }^{2}$-method is more pronounced than in the fine grid search case. The fact that it performs so well in a crude grid search suggests that the PEWS ${ }^{2}$ method is especially suited to efficiently make a first rapid selection when a large number of different combinations of stationary phase, organic modifier, $\mathrm{pH}$, and temperature need to be scouted. Similar conclusions can be made when looking at the average value of the quality criterion $n_{\mathrm{p}}{ }^{*}$ of the best solution found by the search algorithms (Figure S-4 in the Supporting Information). Changing the number of assumed theoretical plates from $N=20000$ to smaller $(N=5000$ and 10000$)$ or larger $(N=40000)$ did not change the relative success rate of the two methods.

Overall, it can be concluded that the advantage of the PEWS $^{2}$-method is that it automatically zooms in on the most promising areas in the search space. This is illustrated in Figure 3 , where a side-by-side comparison is made of the $n_{\mathrm{p}}{ }^{*}$-contour plots in the $\left(\phi_{0}, \beta t_{0}\right)$-domain and those in the $\left(k_{\text {first }}, k_{\text {last }}\right)$-domain for two (randomly selected) different 15 component samples. Some other examples with varying sample complexity $(10,20$, and 25 components) are given in Figure S-5 in the Supporting Information. In each case, the areas corresponding to the highest separation quality are enlarged when going from the $\left(\phi_{0}, \beta t_{0}\right)$-domain to the $\left(k_{\text {first }} k_{\text {last }}\right)$-domain.

Some Real-Life Examples. Separation of an Antiepileptic Drug and Its Impurities. In this first example, the purpose was to determine the best possible linear gradient program for the separation of the API and its 13 impurities after first selecting the best of two proposed stationary phases (T3 and PFP column). The impurities were available in two sets (respectively, containing 9 and 4 impurities); hence, the method needed to be developed for the overlay-chromatogram of both samples. First a column screening has been performed by conducting two initial runs (from 5 to $95 \%$ ACN $+0.1 \%$ TFA in 5 and $15 \mathrm{~min}$ ) on both columns; both on the entire sample (PEWS ${ }^{2}$-method) as well as for the individual compounds (needed for the comparison with Drylab). Subsequently, eight runs (representing different elution windows, see Table S-2 in the Supporting Information for details of selected $k$-values) proposed by the PEWS ${ }^{2}$-method on the basis of the overlay chromatogram were run on each column. Evaluation of these runs showed that the best separations were performed on the T3 column. Fine tuning of the separation on this column (by carrying out eight extra 

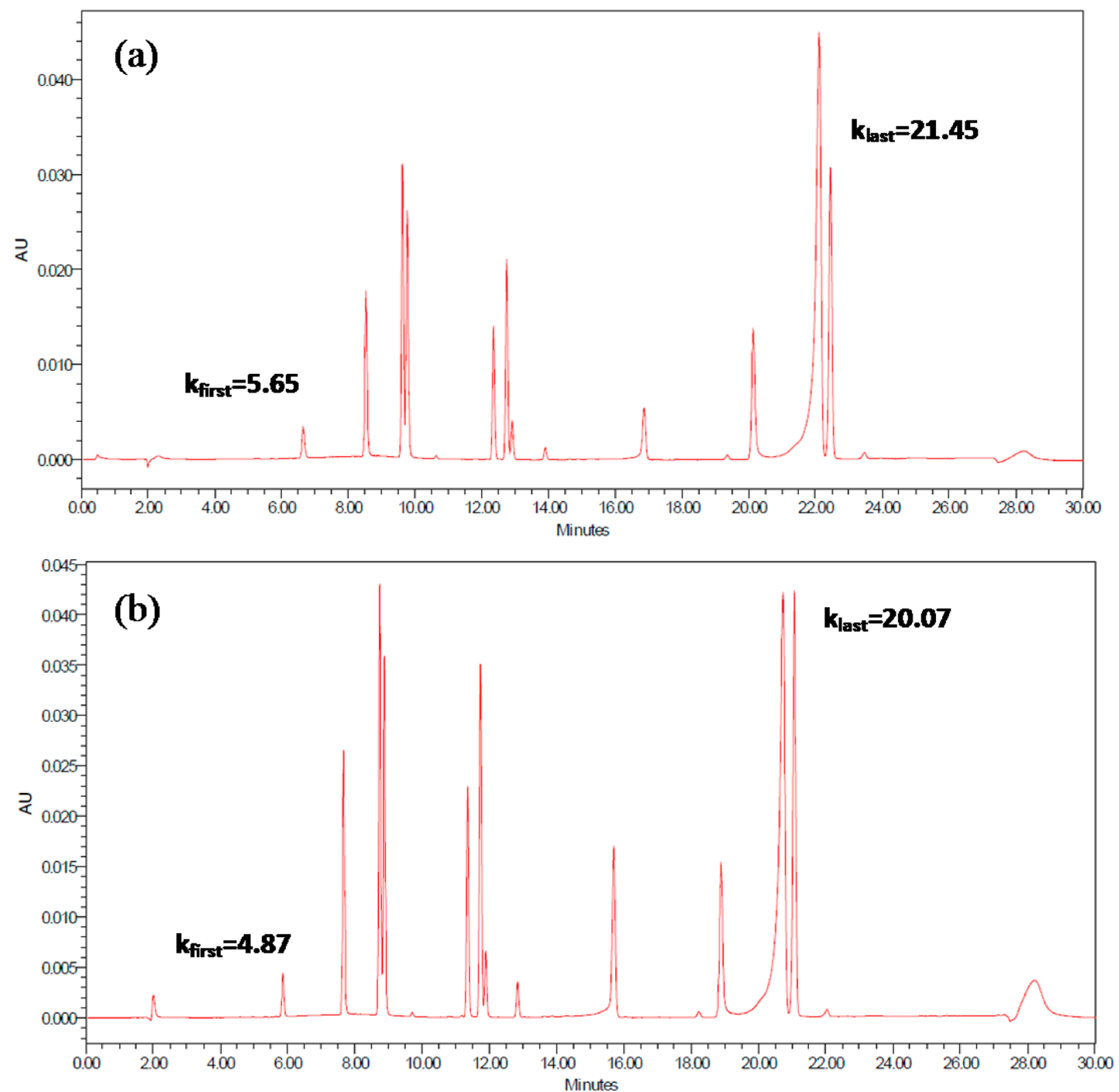

Figure 4. Chromatograms obtained for the separation of the 13 component mixture (a) PEWS ${ }^{2}$ best run (24.2-75.8\% ACN in 25.00 min) and (b) Drylab best run $(20.0-80.0 \% \mathrm{ACN}$ in $25.00 \mathrm{~min})$.

runs configured in a square around the best initial search runs in the $\left(k_{\text {first }} k_{\text {last }}\right)$-space) led to an increased resolution of the critical pairs. The final method, with which all the impurities can be separated from the API, consisted of running a gradient from 7 to $34.3 \% \mathrm{ACN}+0.1 \%$ TFA in a gradient time of 15.37 min (see Figure S-6 in the Supporting Information). To achieve this, the PEWS ${ }^{2}$-method needed 28 runs on the two columns, which could be effectuated in only $12 \mathrm{~h}$, including the column equilibration runs.

The final method proposed by Drylab (using the predicted $k_{\mathrm{w}}$ and $S$ values of all individually available components) was a gradient running from 5 to $34 \% \mathrm{ACN}+0.1 \%$ TFA in $19 \mathrm{~min}$, very similar to the final method obtained by the PEWS $^{2}$ method.

Separation of a 13 Component Pharmaceutical Mixture. In a second example, it was the aim to develop a method for the separation of a 13 pharmaceutical component mixture. First, two preruns (from $5 \%$ to $95 \% \mathrm{ACN}$ in $20 \mathrm{~min}$ and $60 \mathrm{~min}$ ) were carried out to estimate the $k_{\mathrm{w}}$ and $S$ value of all components (used by Drylab) or of the first and last eluting compound only (used by the PEWS ${ }^{2}$-method). Using the PEWS $^{2}$-method to propose eight runs with widely differing elution windows (see Table S-2 in the Supporting Information), it was found that, upon conducting them, all 13 components were revealed during four of these eight initial runs (effectuated in $7 \mathrm{~h}$, including the column equilibration runs). Again, the run with the highest resolution (with parameters $\phi_{0}=0.242, \phi_{\mathrm{e}}=0.758$ and $t_{\mathrm{G}}=25.00 \mathrm{~min}$, see Figure $4 \mathrm{a})$ was very similar to the Drylab solution $\left(\phi_{0}=0.200\right.$, $\phi_{\mathrm{e}}=0.800$, and $t_{\mathrm{G}}=25.00 \mathrm{~min}$, see Figure $\left.4 \mathrm{~b}\right)$. A fine-tuning round did not lead to any improvement in resolution.

Separation of a 16 Component Antipsychotic Drugs Mixture. In the third considered example, it was the purpose to find the best combination of stationary phase and $\mathrm{pH}$ for the separation of a 16 antipsychotic drugs sample. The screening involved four stationary phases (BEH C18, BEH C18 Shield, 
CSH PFP, and BEH Phenyl) and three pHs (3.0, 5.0, and 9.0). First, two preruns (gradient from 5 to $95 \% \mathrm{ACN}$ in 4 and 12 $\mathrm{min}$ ) were carried out on each stationary phase $/ \mathrm{pH}$ combination to determine the $k_{w^{-}}$and $S$-values of the first and the last eluting component for each of these combinations. Any change in elution order was neglected, not the least because the elution order could not be tracked since only UVdetection was used (similar UV spectra for all compounds) and since the components were always injected together. Subsequently, five different gradient conditions (representing different elution windows) were proposed using the PEWS ${ }^{2}$ method for every screening combination. From this initial search, two screening conditions (BEH C18 Shield $\mathrm{pH}=5.0$ and $\mathrm{CSH}$ PFP $\mathrm{pH}=5.0)$ were identified wherein all 16 components were already revealed (see Figure S-7a in the Supporting Information for the separation with the CSH PFP phase). Subsequently, a fine-tuning step was performed for both stationary phase $/ \mathrm{pH}$ combinations. In this step, the PEWS $^{2}$-method was slightly modified by shifting the third (instead of the first) peak of the chromatogram to improve the resolution of the critical pairs even further (peaks 3 and 4, 6 and 7, 8, and 9, and 15 and 16). Doing so, the resolution of the last critical pair could be significantly improved on the Acquity $\mathrm{CSH}$ PFP column (see Figure S-7b in the Supporting Information). The resulting gradient conditions were subsequently performed on a longer column length, by coupling two $50 \mathrm{~mm}$ columns in series (connection volume of $1 \mu \mathrm{L}$ ) in order to further improve the resolution of the resulting critical pairs (peaks 3 and 4, peaks 8 and 9, and peaks 15 and 16). As can be seen in Figure S-7c in the Supporting Information, this leads to a method wherein a nearly complete baseline separation is obtained for all peaks. In total, 84 runs were effectuated (on the different columns and for the different $\mathrm{pHs}$ ), keeping the total run time (including the column reequilibration runs) to do the screening below $12 \mathrm{~h}$.

\section{CONCLUSIONS}

Gradient optimization methods based on the predictive shifting and stretching of the elution window constitute a promising intermediate between the pure search-based and model-based methods. Compared to model-based search-methods, the method has the advantage that the retention properties of the sample compounds do not need to be known or all modeled, such that it can be applied to samples with unknown composition and allowing us to save the time and cost needed to collect the model data (cf. the need for MS-spectra, for example). Compared to pure search-based methods, the algorithm has the advantage that it does not search blindly in the $\left(\phi_{0}, \beta t_{0}\right)$-space but directly in the physically much more relevant $\left(k_{\text {first }}, k_{\text {last }}\right)$-space. Searching in the latter provides a direct zoom-in on the most promising areas of the solution space. This strongly increases the probability to find the best possible solution. The algorithm does not stand or fall with a rigorous knowledge of the retention parameters of all compounds (and is therefore also directly applicable to samples whose composition is not entirely known) or does not run the risk of converging to a false minimum (as is the case with pure search-based methods such as the grid-search or the Simplex method). As such, the proposed algorithm offers a generic "midway" approach between purely model-based and purely search-based algorithms, with many additional possibilities, for example, by changing or extending the number of peaks whose position is fixed in the chromatogram during the course of the algorithm.

Since the PEWS ${ }^{2}$-method does not rely on the prediction of the complete separation resolution (for which the retention times of all individual components need to be predicted exactly), but only on the prediction of the retention times of the first and last components, it is much less sensitive to modeling errors than the pure model-based search methods. This is because the modeling is only used to propose a new search point, where the achieved resolution is anyhow still determined experimentally. When the prediction of the gradient conditions needed to position the elution window at a given position in the chromatogram fails because of changes in elution order at either the beginning or the end of the elution window, a limited form of peak tracking on the first three or four eluting compounds and on the last three or four eluting compounds can be done to continuously update the modeling information for the first and last eluting compounds. If needed, the identity and the number of peaks that are fixed and optimized can be changed during the course of the search, for example, when a problematic zone shows up (as was the case in the 16 component antipsychotic drugs mixture example).

The drawback of the PEWS ${ }^{2}$-method is that it remains a search-based method and therefore always has the inherent risk of missing the best solution (although this risk can be minimized using more advanced search methods than the one used in the present study). How this compares to the risk for small modeling errors of the pure model-based methods (leading to a wrongly proposed solution in the case of a crowded chromatogram) is difficult to quantify and generalize.

The convergence properties of this new MD strategy have been evaluated by applying it to three different real-world separation problems, which could all be solved successfully in a very short time (on the order of 1 or 2 days of instrument time). Making a direct numerical comparison test based on 1000 different in silico samples with realistic retention properties, it was found that the $\mathrm{PEWS}^{2}$-algorithm could reduce the number of missed components by about $50 \%$ and more. Compared to the pure grid-based search, the method on the average also produces solutions that lie a few percent (order of $1-2 \%$ ) closer to the highest achievable separation quality number $n_{\mathrm{p}} *$ than the pure-grid based search. The advantage of the PEWS ${ }^{2}$-algorithm was found to grow with increasing sample complexity and decreasing available search time. The latter also implies that the algorithm is especially suited to efficiently make a first rapid selection among a large number of different combinations of stationary phase, organic modifier, $\mathrm{pH}$, and temperature.

Future efforts will focus on combining the algorithm with more powerful search techniques (using for example the information of the slope on the different parts of the response surface that can be established in the course of the search process to enhance the success rate of the search) as well as on the extension of the method to multistep gradients (which could be by done by fixing the retention factors of the peaks in the most problematic zone(s) of the chromatogram instead of only fixing the first and last peaks of the entire chromatogram as was done in the present study).

\section{ASSOCIATED CONTENT}

\section{S Supporting Information}

Additional information as noted in text. This material is available free of charge via the Internet at http://pubs.acs.org. 


\section{AUTHOR INFORMATION}

\section{Corresponding Author}

*E-mail: gedesmet@vub.ac.be.

\section{Notes}

The authors declare no competing financial interest.

\section{ACKNOWLEDGMENTS}

E.T. gratefully acknowledges a grant from the Research Foundation-Flanders (FWO).

\section{REFERENCES}

(1) de Villiers, A.; Kalili, M.; Malan, M.; Roodman, J. LC-GC Eur. 2010, 23 (10), 466-478.

(2) Jandera, P. J. Chromatogr., A. 2006, 1126, 195-218.

(3) Dolan, J. W. LC-GC Eur. 2010, 23 (12), 581-584.

(4) Dolan, J. W. LC-GC Eur. 2011, 24 (1), 20-24.

(5) Pursh, M.; Scheiwer-Theobaldt, A.; Cortes, H.; GratzfeldHuesgen, A.; Schulenberg-Shell, H.; Hoffmann, B.-W. LC-GC Eur. 2008, 21 (3), 152-159.

(6) De Beer, M.; Lynen, F.; Chen, K.; Ferguson, P.; Hanna-Brown, M.; Sandra, P. Anal. Chem. 2010, 82, 1733-1743.

(7) Goga, S.; Heinisch, S.; Rocca, J. L. Chromatographia 1998, 48, 237-244.

(8) Nikitas, P.; Papa-Louisi, A. J. Liq. Chromatogr. Relat. Technol. 2009, 32, 1527-1576.

(9) Dharmadi, Y.; Gonzalez, R. J. Chromatogr., A. 2005, 1070, 89101.

(10) Giddings, J. M.; Davis, J. C. Anal. Chem. 1985, 57, 2168-2177.

(11) Berridge, J. C. J. Chromatogr. 1982, 244, 1-14.

(12) Berridge, J. C.; Morrisey, E. G. J. Chromatogr. 1984, 316, 69-79.

(13) Dolan, J. W.; Lommen, D. C.; Snyder, L. R. J. Chromatogr. 1989, $485,91-112$.

(14) Galushko, S. V.; Kamenchuk, A. A.; Pit, G. L. J. Chromatogr., A. 1994, 660, 47-59.

(15) I, T. P.; Smith, R.; Guhan, S.; Taksen, K.; Vavra, M.; Myers, D.; Hearn, M. T. W. J. Chromatogr., A 2002, 972, 27-43.

(16) Martinez-Vidal, J.-L.; Parrilla, P.; Fernandez-Alba, A. R.; Carreno, R; Herrera, F. J. Liq. Chromatogr. 1995, 18, 2969-2989.

(17) Berridge, J. C. J. Chromatogr. 1989, 485, 3-14.

(18) Molnar, I. J. Chromatogr., A 2002, 965, 175-194.

(19) Hewitt, E. F.; Lukulay, P.; Galushko, S. J. Chromatogr., A. 2006, 1107, 79-87.

(20) Concha-Herrera, V.; Vivó-Truyols, G.; Torres-Lapasió, J. R.; Garcia-Alvarez-Coque, M. C. J. Chromatogr., A 2005, 1063, 79-88.

(21) Nikitas, P.; Papa-Louisi, A. Anal. Chem. 2005, 77, 5670-5677.

(22) Jupille, T.; Snyder, L. R.; Dolan, J. W. LC-GC Eur. 2002, 15 (9), 596-601.

(23) Shan, Y.; Weibing, Z.; Seidel-Morgenstern, A.; Ruihuan, Z.; Yukui, Z. Sci. China, Ser. B: Chem. 2006, 49, 315-325.

(24) Morgan, S. L.; Deming, S. N. J. Chromatogr. 1975, 112, 267285.

(25) Watson, M. W.; Carr, P. W. Anal. Chem. 1979, 51, 1835-1842.

(26) Djordevic, N. M.; Erni, F.; Schreiber, B.; Lankmayr, E. P.; Wegscheider, W.; Jaufman, L. J. Chromatogr. 1991, 550, 27-37.

(27) Torrés-Lapasio, J. R.; Garcia-Alvarez-Coque, M. C.; Bosch, E.; Rosés, M. J. Chromatogr., A 2005, 1089, 170-186.

(28) Duarte, R. M. B. O.; Duarte, A. C. J. Chromatogr., A 2010, 1217, $7556-7563$.

(29) Ortín, A.; Torres-Lapasio, J. R.; Garcia-Alvarez-Coque, M. C. J. Chromatogr., A. 2011, 5829-5836.

(30) Neue, U. D.; Kuss, H.-J. J. Chromatogr., A 2010, 1217, 37943803.

(31) Snyder, L. R. J. Chromatogr. 1964, 11, 415-434.

(32) Snyder, L. R., Dolan, J. W. High-Performance Gradient Elution: The Practical Application of the Linear Solvent Strength Model; Wiley Interscience: Hoboken, NJ, 2007.
(33) Schoenmakers, P. J.; Billiet, H. A. H.; Tijssen, R.; De Galan, L. J. Chromatogr. 1978, 149, 519-537.

(34) Guiochon, G., Shirazi, S. G., Katti, A. M. Fundamentals of Preparative and Nonlinear Chromatography; Academic Press, 1994.

(35) Neue, U. D. HPLC Columns: Theory, Technology and Practice; Wiley-VCH: New York, 1997.

(36) Neue, U. D.; Marchand, D. H.; Snyder, L. R. J. Chromatogr., A 2006, 1111, 32-39. 